\title{
Xyloglucan Structure Impacts the Mechanical Properties of Xyloglucan-Cellulose Nanocrystal Layered Films-A Buckling-Based Study
}

Taylor C. Stimpson, Bernard Cathala, Céline Moreau, Jose M. Moran-Mirabal,** and Emily D. Cranston*

Cite This: Biomacromolecules 2020, 21, 3898-3908

Read Online

ABSTRACT: Interactions between polysaccharides, specifically between cellulose and hemicelluloses like xyloglucan (XG), govern the mechanical properties of the plant cell wall. This work aims to understand how XG molecular weight (MW) and the removal of saccharide residues impact the elastic modulus of $\mathrm{XG}$-cellulose materials. Layered sub-micrometer-thick films of cellulose nanocrystals (CNCs) and XG were employed to mimic the structure of the plant cell wall and contained either (1) unmodified XG, (2) low MW XG produced by ultrasonication (USXG), or (3) XG with a reduced degree of galactosylation (DGXG). Their mechanical properties were characterized through thermal shrinking-induced buckling. Elastic moduli of $19 \pm 2,27 \pm 1$,
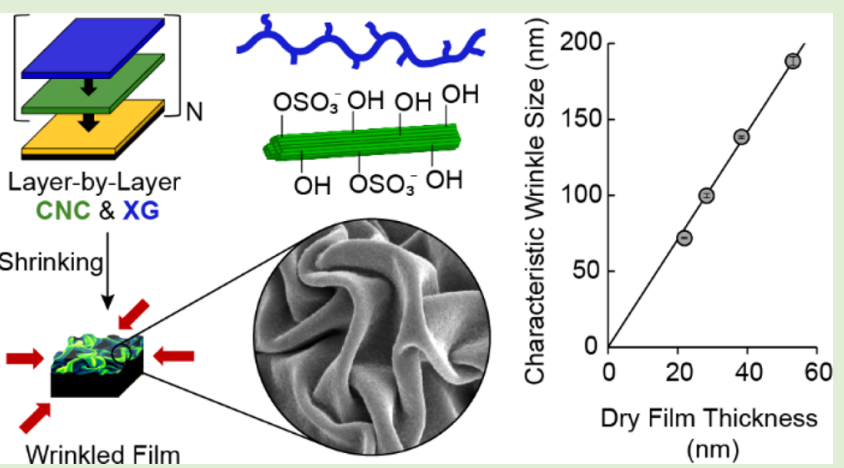
and $75 \pm 6 \mathrm{GPa}$ were determined for XG-CNC, USXG-CNC, and DGXG-CNC films, respectively. The conformation of XG adsorbed on CNCs is influenced by MW, which impacts mechanical properties. To a greater degree, partial degalactosylation, which is known to increase XG self-association and binding capacity of XG to cellulose, increases the modulus by fourfold for DGXG-CNC films compared to XG-CNC. Films were also buckled while fully hydrated by using the thermal shrinking method but applying the heat using an autoclave; the results implied that hydrated films are thicker and softer, exhibiting a lower elastic modulus compared to dry films. This work contributes to the understanding of structure-function relationships in the plant cell wall and may aid in the design of tunable biobased materials for applications in biosensing, packaging, drug delivery, and tissue engineering.

\section{INTRODUCTION}

The primary plant cell wall plays a key role in plant function including structural support, intercellular communication, control of cell growth, and barrier protection. ${ }^{1,2}$ The plant cell wall is composed of an intricate polysaccharide network that possesses remarkable mechanical properties. This network offers the plant flexibility, allowing expansion during cell growth, while providing structural support to retain cellular integrity and rigidity. ${ }^{3}$ Such properties have inspired researchers to both investigate relationships between cell wall structure and function ${ }^{1}$ and design new materials composed of, or mimicking, plant cell wall components. ${ }^{4-11}$ Bioinspired plant cell wall materials may offer biobased and environmentally benign solutions for applications in coatings, composites, hydrogels, and packaging, to name a few. In this work, plant cell wall mimics were designed and probed to better understand structure-function relationships related to mechanical properties.

The plant cell wall is a composite material made of cellulose fibrils embedded in a matrix of hemicellulose, lignin, pectin, proteins, and water. ${ }^{12,13}$ Cellulose, which makes up $20-30 \%$ of the cell wall (by dry mass), ${ }^{13,14}$ is a high-molecular weight
(MW) biopolymer composed of $\beta$-1,4-linked D-glucose units. ${ }^{2}$ Adjacent cellulose chains interact to form a hierarchical structure of tightly bound elementary fibrils bundled into microfibrils. ${ }^{15}$ Elementary cellulose fibrils have highly ordered (tightly packed) crystal regions interspersed with less-ordered cellulose regions. ${ }^{16}$ The cellulose microfibrils and their orientation angle define the major load-bearing structure of the plant cell wall, where the crystalline regions are reported to have an average transverse elastic modulus of $\sim 140 \mathrm{GPa}^{15}$ Thus, cellulose has an elastic modulus similar to that of Kevlar $(60-125 \mathrm{GPa})$ and close to that of steel (200-220 GPa), while having a much lower density than most engineering materials (e.g., $1.5-1.6 \mathrm{~g} / \mathrm{cm}^{3}$ for cellulose compared to $8 \mathrm{~g} /$ $\mathrm{cm}^{3}$ for steel). ${ }^{15,17}$ The impressive mechanical properties of

Received: July 7, 2020

Revised: August 13, 2020

Published: August 17, 2020 
cellulose make it an interesting structural support material for engineering composites.

Hemicellulose is the second most important material in the plant cell wall in terms of structural support, and similar to cellulose, it is characterized by having a $\beta$-1,4-linked sugar backbone. $^{2,18}$ However, hemicelluloses are a heterogeneous group of polysaccharides ${ }^{18}$ and have different repeating disaccharides of 5- and 6-carbon sugars, specifically glucose, mannose, and xylose. ${ }^{16,18}$ In addition, unlike the linear chains ${ }^{19}$ of cellulose, hemicellulose is branched, where branch residues vary for hemicellulose from different plant sources. Xyloglucan (XG) is among the most common hemicelluloses found in the cell wall of terrestrial plants and comprises $2-25 \%$ (by dry mass). ${ }^{18,20} \mathrm{XG}$ has a backbone of $\beta$-1,4-linked glucans which is substituted with a regular pattern of $\alpha-1,6$-xylosyl residues. The xylose units can be substituted with $\beta$-1,2-linked galactosyl residues, and some XGs are further substituted with arabinose or fucose. ${ }^{18,21,22} \mathrm{XG}$ is known to bind to cellulose fibrils to form tethers or cross-links, forming a mechanically strong but flexible network and serving as the main mechanical structure of the cell wall. ${ }^{21-25}$ The exact nature of XG-cellulose interactions is still a subject of ongoing research, but it is generally accepted that the adsorption of XG to cellulose is entropically driven (through the release of water molecules) and the structures are held together through van der Waals forces and hydrogen bonding. ${ }^{26}$ Recent studies highlight the mechanical significance of the XG-cellulose network, noting that "biomechanical hotspots" are present where XG directly binds to cellulose. ${ }^{24}$ The biomechanical hotspot concept, reviewed by Park and Cosgrove, ${ }^{27}$ refers to the interlacing of small portions of XG with cellulose microfibrils, which result in tight mechanical junctions between the microfibrils that are relatively inaccessible to enzymatic degradation.

Previous work has employed plant cell wall mimics to better understand the role of the XG structure on its capacity to bind to cellulose. ${ }^{4-8,26,28,29}$ Specifically, XG has been modified by altering the MW or degree of substitution of different saccharide residues through physical, chemical, or enzymatic treatments, and the adsorption to model cellulose films has been quantified. ${ }^{26,29-31}$ It was found that at low MW, XG forms extended conformations on the surface of cellulose, forming closely packed sandwich structures between cellulose fibrils. ${ }^{32,33}$ Conversely, high MW XG tends to form "loops and tails" between cellulose, which increases matrix swelling during hydration and is more accessible to enzymatic degradation. ${ }^{32,33}$ XG saccharide residues are known to affect XG self-association and adsorption to cellulose. ${ }^{29,34,35}$ The amount of fucosyl and galactosyl residues of XG were altered by selective enzymatic degradation, and while removal of fucosyl residues did not have a significant impact on adsorption to cellulose, removal of galactose groups (by 20-50\%) resulted in strong selfassociation of $\mathrm{XG}$, forming a reversible, thermoresponsive gel. $^{35,36}$ XG with fewer galactose groups also showed a higher mass adsorption onto cellulose than unmodified XG, forming a more compact structure. ${ }^{29,34-36}$

Past work on modifying XG structure highlights its importance on the ability of XG to adsorb to, and form a network with, cellulose. However, the effect of the XG structure on mechanical properties of the plant cell wall or mimic materials is yet to be explored. Here, sub-micrometerthick films were constructed from cellulose nanocrystals (CNCs) and XG with varied $\mathrm{MW}$ and degree of galactosylation. CNCs are rigid rod-shaped nanoparticles often used as model cellulose surfaces, ${ }^{37,38}$ especially in combination with XG. ${ }^{25,28,32,36,39-41}$ CNCs can be isolated using sulfuric acid hydrolysis of natural cellulose (e.g., wood pulp, cotton, agricultural waste, and microbial cellulose). ${ }^{42}$ CNCs from plants have typical lengths of $100-200 \mathrm{~nm}$ and cross sections of 5-10 nm, depending on their exact source and method of extraction, ${ }^{43,44}$ and have a high degree of crystallinity (54-99\%). ${ }^{43-45}$ CNCs are a good model for native cellulose because they are made solely of cellulose and have similar crystalline and mechanical properties to cellulose microfibrils but are easier to process to form smooth films of uniform thickness. ${ }^{46}$

Our lab has previously demonstrated a thermally induced buckling-based method to compare the mechanical properties of XG-CNC films with polyethylene imine (PEI, a branched cationic polyelectrolyte)-CNC thin films. ${ }^{8,47}$ In this method, a CNC composite film is deposited onto a prestressed polystyrene (PS) substrate that shrinks upon heating above its glass transition temperature $\left(T_{\mathrm{g}}\right)$. The composite film, which has a higher elastic modulus than the PS, buckles to dissipate strain as the substrate shrinks, resulting in a wrinkled morphology. The elastic modulus of the composite is calculated from the wrinkle size and the film thickness. ${ }^{8}$ This method circumvents limitations of other approaches ${ }^{10}$ used to evaluate the mechanical properties of thin films, such as the need for free-standing films, the ability to perform only localized measurements, or the susceptibility to environmental humidity conditions. An additional advantage is that this buckling method offers high-throughput characterization with high precision and low sensitivity to small variations in experimental factors, ${ }^{47}$ which enables the rapid measurement of multiple samples.

In this work, we compared the elastic moduli of XG-CNC thin films composed of unmodified XG or XG with modified MW or degree of galactosylation. Thin films $(<\mu \mathrm{m})$ were constructed using the layer-by-layer (LbL) deposition technique, ${ }^{48}$ resulting in alternating layers of XG and CNCs. ' The films were thermally shrunk, to extract elastic moduli based on buckling mechanics. In addition, an alternate autoclave shrinking method is presented, for the first time, to examine the effect of hydration during buckling. XG-CNC films allow us to extrapolate how the structural modifications of XG affect mechanical properties of the plant cell wall and suggest routes to tailor the mechanical properties of biobased materials for upcoming applications.

\section{EXPERIMENTAL SECTION}

Materials. Prestressed PS shrink films (Graphix Shrink Film, Maple Heights, OH, USA) were used as substrates for the thin films. XG was extracted from Tamarindus indica, containing $90 \%$ XG and 10\% glucose (GLYLOID 6C, Batch 17.01.27; DSP Gokyo Food \& Chemical Co., Ltd. Osaka, Japan). $\beta$-Galactosidase from Aspergillus oryzae (11.8 U/mg, G5160-125KV Batch: SLBS1891V, SigmaAldrich, St. Louis, MO, USA) was used for enzymatic degalactosylation of XG. Spray-dried CNCs in the neutralized sodium form were kindly provided by CelluForce (CelluForce NCC, Batch 2015-009, CelluForce, Canada). CelluForce CNCs (from the same batch as those used in this study) have a surface charge of $\sim 250 \mu \mathrm{mol} \mathrm{OSO}_{3}{ }^{-}$/ g CNC, zeta potential of $-40 \mathrm{mV}$, average length of $183 \pm 88 \mathrm{~nm}$, cross-section of $6 \pm 2 \mathrm{~nm}$, and aspect ratio of 31 as measured by atomic force microscopy, and $89-90 \%$ crystallinity measured by X-ray diffraction. ${ }^{43}$ Polyallylamine hydrochloride (PAH, MW 120,000$200,000 \mathrm{~g} / \mathrm{mol}$ ) was purchased from Polysciences (Warrington, PA, USA). Gold (99.999\% purity, LTS Chemical Inc., Chestnut Ridge, 
NY, USA) was used for calibration of elastic modulus equations. Si wafers used as substrates were purchased from University Wafers ( Ntype, $\langle 100\rangle$ orientation, University Wafers, South Boston, MA, USA) and cleaned with piranha solution (3:1 solution of $\mathrm{H}_{2} \mathrm{SO}_{4} / \mathrm{H}_{2} \mathrm{O}_{2}$ ). All solutions were made using ultrapure Milli-Q water (Milli-Q A10 Purification System, Millipore, Etobicoke, Canada).

Material Preparation. PS Substrate Preparation. Prestressed PS sheets were cut into $2 \mathrm{~cm} \times 2 \mathrm{~cm}$ square substrates with a ROBO Pro CE5000-40-CRP cutter (ROBO Pro CE5000-40-CRP, Graphtec America Inc., Irvine, CA, USA) with the CB15UB ceramic blade (Graphtec America Inc., Irvine, CA, USA) using parameters of 30, 1, and 1 , for force, quality, and speed, respectively. The cut substrates were cleaned in consecutive baths of isopropanol, ethanol, and ultrapure water for $5 \mathrm{~min}$ each under $60 \mathrm{rpm}$ of orbital agitation. The substrates were then dried with compressed nitrogen and stored until use.

Preparation of CNC Suspensions. Powdered CelluForce CNCs (as received in sodium form) were redispersed in ultrapure water at 3 wt \% by first slowly adding CNC powder to a beaker of water and stirring for $>1 \mathrm{~h}$. Once no visible aggregates were observed in the CNC suspension, they were point probe-sonicated (Sonifier 450, Branson Ultrasonics, Danbury, CT, USA) for three rounds of $30 \mathrm{~s}$ intervals with $1 \mathrm{~min}$ cooling between rounds at a $60 \%$ maximum amplitude (120 W input power), while immersed in an ice bath (to prevent temperature of the suspension exceeding $\left.60{ }^{\circ} \mathrm{C}\right) .{ }^{49}$

$X G$ Fractionation. Desired concentrations of $\mathrm{XG}$ in solution were produced by dissolution of $\mathrm{XG}$ in ultrapure water at $50{ }^{\circ} \mathrm{C}$ for $2 \mathrm{~h}$ and then at ambient temperature overnight. XG was stored in a sealed container at $4{ }^{\circ} \mathrm{C}$ until use. To understand how MW and galactose residue content affect $\mathrm{XG}-\mathrm{CNC}$ interactions, $\mathrm{XG}$ fractions with decreased MW and degree of galactosylation were prepared via ultrasonication and enzymatic treatment, respectively.

Ultrasonication of $X G$. Tamarind seed XG was subjected to ultrasonic treatment to obtain XG with a reduced molar mass. ${ }^{32,33}$ Ultrasonic treatment was performed on $800 \mathrm{~mL}$ (separated into $4 \times$ $200 \mathrm{~mL}$ batches) of a $1 \mathrm{wt} \%$ solution of XG using a QSonica Q700 probe sonication device (Q700, $230 \mathrm{~V}, 700 \mathrm{~W}, 20 \mathrm{kHz}$; Qsonica, Newtown, CT, USA). Sonication was performed for $40 \mathrm{~min}$ at a maximum amplitude of $25 \%$ ( 8 rounds of $5 \mathrm{~min}$ ) with the sample submerged in an ice bath to prevent sample degradation from overheating. To remove small fragments, the ultrasonicated XG (USXG) was dialyzed (Spectrum Labs Spectra/Por $33.5 \mathrm{kD}$ MWCO Standard Regenerated Cellulose Dry Dialysis Tubing, Fisher Scientific, UK) in a large container of ultrapure water for 10 days, with water changed twice daily to increase osmotic pressure and therefore mass transfer efficiency. Purified USXG was frozen in 100 $\mathrm{mL}$ aliquots at $-20^{\circ} \mathrm{C}$ and subsequently freeze-dried and stored in a sealed container at ambient temperature until use.

Degalactosylation of XG. Degalactosylation of XG involves the enzymatic removal of galactose residues that decorate the backbone of $\mathrm{XG}$, resulting in partially degalactosylated XG (DGXG). ${ }^{35}$ A buffer solution was first prepared by dissolving $4.5 \mathrm{~g}$ of $\mathrm{KH}_{2} \mathrm{PO}_{4}$ in $500 \mathrm{~mL}$ ultrapure water and adjusting the $\mathrm{pH}$ to $4.8-5$ using $6.25 \mathrm{M}(25 \%)$ $\mathrm{HCl}$, subsequently adding an additional $300 \mathrm{~mL}$ of ultrapure water. A sample $(11 \mathrm{~g})$ of unmodified tamarind seed XG was added to the buffer solution at $50{ }^{\circ} \mathrm{C}$ for $2 \mathrm{~h}$ and then overnight at ambient temperature, giving a solution with final concentrations of 5.63 and $13.75 \mathrm{~g} / \mathrm{L}$ of $\mathrm{KH}_{2} \mathrm{PO}_{4}$ and $\mathrm{XG}$, respectively.

The degalactosylation reaction was initiated by dissolving $350 \mathrm{mg}$ of $\beta$-galactosidase from $A$. oryzae in $50 \mathrm{~mL}$ of ultrapure water, while the XG/buffer solution was heated in a round-bottom flask submerged in an oil bath to reach a solution temperature of $50{ }^{\circ} \mathrm{C}$. The $\beta$-galactosidase solution was added to the heated $\mathrm{XG} / \mathrm{buffer}$ solution; the round-bottom flask was capped with a rubber stopper and allowed to react for $24 \mathrm{~h}$. At $24 \mathrm{~h}$, the solution was immediately transferred to an ice bath to cool the DGXG to $4{ }^{\circ} \mathrm{C}$, inhibiting any further enzymatic activity.

The DGXG solution was then precipitated in $400 \mathrm{~mL}$ batches into $1.2 \mathrm{~L}$ of ethanol, and the water was manually pressed out of the DGXG. After performing vacuum filtration on the DGXG, the material was rinsed once more in ethanol and then vacuum-filtered again. The material was precipitated into ether, and one final vacuum filtration was performed. The purified sample of DGXG was dried in a crystallization dish in a fume hood at ambient temperature and stored under ambient conditions until use. In our previous work, monosaccharide composition analysis was performed on DGXG and measured by gas-liquid chromatography, indicating a galactose removal ratio of $49 \%$ (a reduction of galactose content from $\sim 16 \%$ dry weight of unmodified XG to $\sim 8 \%$ dry weight of DGXG) ${ }^{36}$ For use in solution, samples were dissolved at the desired concentration at $4{ }^{\circ} \mathrm{C}$ for $24 \mathrm{~h}$. DGXG solutions were stored in sealed containers at 4 ${ }^{\circ} \mathrm{C}$.

Plant Cell Wall Mimic Film Construction. LbL Assembly. Layered films of CNCs and XG (unmodified and modified) were constructed using aqueous-based dip coating with the LbL method. ${ }^{7}$ Film thickness was controlled by the number of deposition steps during dip coating. Films were deposited onto either PS or $\mathrm{Si}$ wafer substrates for shrinking and thickness characterization, respectively. Clean PS or Si substrates were first treated using air plasma at 600 mTorr for $3 \mathrm{~min}$ (PDC001 Expanded Plasma Cleaner, Harrick Plasma, Ithaca, NY, USA) to increase wettability. The treated substrates were then dipped into a $1 \mathrm{wt} \%$ solution of PAH for $15 \mathrm{~min}$ to act as an anchoring layer and improve adhesion between the film and the PS substrate. The samples were rinsed in ultrapure water for 10 min to remove any loosely bound polymer and dried with a stream of compressed nitrogen.

Following deposition of the $\mathrm{PAH}$ layer, alternating layers of CNCs and either XG, DGXG, or USXG were deposited by dipping the sample in 3 wt \% CNCs and 0.01 wt \% XG/DGXG/USXG for 15 min. In between each layer, a $10 \mathrm{~min}$ rinsing step was performed to remove loosely bound XG or CNCs, followed by drying with compressed nitrogen. This process was repeated with alternating CNCs and XG/DGXG/USXG dipping steps until 3, 5, 7, and 10 bilayers (bL) were deposited, where $1 \mathrm{bL}$ denotes one layer of CNCs and one layer of XG/DGXG/USXG. Samples were stored in Petri dishes at ambient temperature until further used.

Gold Film Preparation. Gold films were used as a material for calibration of elastic modulus equations because of its well-known material properties. ${ }^{50}$ Gold films of $125 \mathrm{~nm}$ thickness were deposited onto PS substrates using a Torr Compact Research Coater CRC-600 manual planar magnetron sputtering system (Torr International, New Windsor, NY, USA) using a deposition rate of $0.5 \AA / \mathrm{s}$. Samples were stored in Petri dishes under ambient conditions until use.

Film Shrinking Methods. Layered XG/USXG/DGXG-CNC films were buckled using two different methods: dry thermal shrinking (in an oven) and hydrated thermal shrinking (in an autoclave, model 2540E Autoclave, Tuttnauer, NY, USA). Gold films were also buckled via both methods for calibration. Thermal dry shrinking was achieved by heating the sample in an oven to $135^{\circ} \mathrm{C}$, above the $T_{\mathrm{g}}$ of PS, for 15 min. Samples in the autoclave were heated for $30 \mathrm{~min}$ at $121{ }^{\circ} \mathrm{C}$ (also above the $T_{\mathrm{g}}$ of PS), $117 \mathrm{kPa}$, and $100 \% \mathrm{RH}$. Times for shrinking were based on achieving "complete" shrinking; samples were fully shrunk when the final size was $40 \%$ of the original (strain $\approx 0.6$ ).

Characterization Methods. High-Performance Size-Exclusion Chromatography. XG, USXG, and DGXG samples were dissolved in $50 \mathrm{mM} \mathrm{NaNO}{ }_{3}$ (99\% Sigma Ultra S8170-250G) at $5 \mathrm{~g} / \mathrm{L}$. The eluent used contained $0.02 \% \mathrm{NaN}_{3}$ in $50 \mathrm{mM} \mathrm{NaNO}$. XG samples were eluted at a rate of $0.6 \mathrm{~mL} / \mathrm{min}$ through a Shodex OHpak SB-805 HQ column $(8 \mathrm{~mm} \times 300 \mathrm{~mm})$. A multiangle laser light scattering detector (mini-Dawn, Wyatt, USA), a differential refractometer (ERC $7517 \mathrm{~A}$ ), and a differential viscometer (T-50A, Viscotek, USA) were used to determine MW and intrinsic viscosity in-line. MWs of XG were determined using the Astra 1.4 software (Wyatt, USA). A $\mathrm{d} n / \mathrm{d} c$ ratio of $0.147 \mathrm{~mL} / \mathrm{g}$ was used to calculate concentrations. Table 1 summarizes the MWs obtained for each variation of XG, as measured in a prior study on the same materials. ${ }^{36}$ We note that in addition to the mass lost as a result of degalactosylation in the DGXG sample, some backbone cleavage occurred as well because of endoglucanase contamination, ${ }^{36}$ making the MW of USXG and DGXG most comparable and significantly lower than unmodified XG. 
Table 1. Weight Average $\left(M_{\mathrm{w}}\right)$ and Number Average MW $\left(M_{n}\right)$ of Unmodified XG, USXG, and DGXG as Measured by HPSEC, Characterized Previously by the Authors ${ }^{36}$

$\begin{array}{ccc}\text { XG variant } & M_{\mathrm{w}}\left(10^{3} \mathrm{~g} / \mathrm{mol}\right) & M_{\mathrm{n}}\left(10^{3} \mathrm{~g} / \mathrm{mol}\right) \\ \text { XG } & 840 & 674 \\ \text { USXG } & 326 & 214 \\ \text { DGXG } & 303 & 224\end{array}$

Scanning Electron Microscopy. Buckled films on PS were prepared for scanning electron microscopy (SEM) imaging by mounting the samples on $1^{\prime \prime}$ stainless steel stubs using carbon tape and nickel paint to establish contact between the stub and sample and reduce charging during imaging. The samples were coated with $7 \mathrm{~nm}$ of platinum using a precision etching coating system (model 682, Gatan Inc., CA, USA). Images were taken using a JEOL 7000F SEM (JEOL, Tokyo, Japan) at an acceleration voltage of $2 \mathrm{kV}$ and working distances between 3 and $6 \mathrm{~mm}$.

Variable Angle Spectroscopic Ellipsometry. Thickness measurements of LbL films deposited on Si wafers were obtained using an M2000UI variable angle spectroscopic ellipsometer (J.A. Woollam, Lincoln, NE, USA). Measurements were taken over a range of wavelengths from 250 to $1680 \mathrm{~nm}$ and incident angles of $55-75^{\circ}$, in $5^{\circ}$ increments. The CompleteEASE software was employed to extract film thickness from ellipsometric data using a Cauchy model to fit the data under the assumption of transparent films on an $\mathrm{Si}$ substrate. Parameters for the fits were confined to positive values, where $A \approx$ $1.5, B<0.02$, and $C<2 \times 10^{-4}$. To ensure film uniformity, thickness was measured on $\geq 3$ spots across the film surface, for $\geq 3$ samples for each number of $\mathrm{bL}$, and the error bars represent the standard deviation of replicate measurements.

Digital Image Processing and Analysis. SEM images were used for digital processing to determine the characteristic wrinkle wavelength of buckled films. Using ImageJ (ImageJ 1.52a, Wayne Rasband, National Institutes of Health, USA), images taken at different magnitudes were cropped to $900 \times 900$ pixels and adjusted for contrast and brightness to improve feature detection. Canny edge detection was performed using ImageJ to detect wrinkle edges. ${ }^{31}$ Fourier transforms, data filtering, and curve fitting were performed using a MATLAB algorithm developed in-house (MATLAB R2014b, MathWorks, USA). ${ }^{52}$ This algorithm was optimized in previous work to accurately extract wrinkle wavelengths of buckled materials. ${ }^{52}$ Fourier analysis and all associated steps described were performed, in triplicate, for all $\mathrm{XG}-\mathrm{CNC}$ films over the range of thickness values, as well as gold films for method calibration (error bars represent standard deviation values from the three measurements).

Calculation of Elastic Moduli. Plots of characteristic wrinkle wavelength $(\zeta)$ as a function of film thickness $(h)$ were used to determine the elastic moduli of the LbL thin films of unmodified and modified XG with CNCs. Data were fit to a linear regression, following the form

$$
\xi=a \zeta=2 \pi \eta^{2 / 3} h
$$

where $a$ is a scaling factor used to relate characteristic wrinkle wavelength $(\zeta)$ to persistence length $(\xi)$. This is used to determine the factor $\eta$, which in turn is used to calculate film elastic modulus $\left(E_{\mathrm{f}}\right)$ following

$$
E_{\mathrm{f}}=3 E_{\mathrm{S}} \frac{\left(1-\nu_{\mathrm{f}}^{2}\right)}{\left(1-\nu_{\mathrm{S}}^{2}\right)} \eta
$$

where $E_{S}$ is the elastic modulus of the substrate at the processing temperature, in this case, PS at $135^{\circ} \mathrm{C}$, and $\nu_{\mathrm{f}}$ and $\nu_{\mathrm{S}}$ are the Poisson ratios for the film and substrate, respectively. The scaling factor, $a$, is calibrated by measuring characteristic wavelengths of thin films of sputtered gold with known thickness and reported Poisson ratio $(0.42)$ and elastic modulus (70 GPa). ${ }^{50,53}$ For the substrate, a Poisson's ratio and elastic modulus of 0.36 and $1.63 \mathrm{GPa}$ were used, respectively, corresponding to PS at $135{ }^{\circ} \mathrm{C} .{ }^{8,54}$ To calculate the moduli of the LbL films, a Poisson ratio of 0.3 is used, which is equivalent to that reported elsewhere for cellulose and hemicellulose. $^{55}$

\section{RESULTS AND DISCUSSION}

Buildup and Characterization of XG-CNC Layered Thin Films. Layered films composed of CNCs and unmodified or modified XG were dip-coated onto prestressed PS and Si wafers for thermal shrinking and thickness measurements, respectively. Films were fabricated on substrates using the well-known aqueous-phase LbL deposition method, ${ }^{56}$ which has been previously used to make XG-CNC composite films. $5,7,8$ The LbL method involved alternating dipping steps of 3 wt \% CNCs and 0.01 wt \% XG, for each variation of $\mathrm{XG}$ examined, with rinsing steps in between (Figure 1A). To study the influence of the XG structure on the

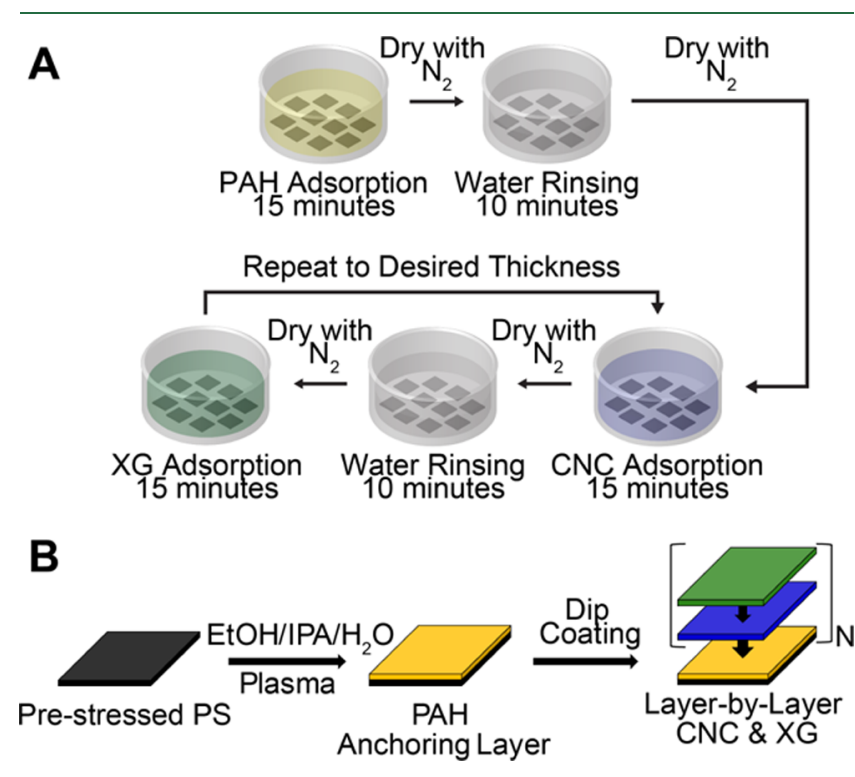

Figure 1. Schematic representation of (A) LbL dip coating and (B) layered structures of XG/DGXG/USXG-CNC films. XG, in the figure, denotes unmodified and modified XG (i.e., XG, USXG, and DGXG).

mechanical properties of layered XG-CNC films, unmodified XG, XG with reduced MW (USXG, via ultrasonication), and $X G$ with a reduced degree of galactosylation (DGXG, $49 \%$ degalactosylated via enzymatic treatment) were used. XGCNC, USXG-CNC, and DGXG-CNC films with 3, 5, 7, and $10 \mathrm{bL}$ were fabricated and characterized, where one bL is defined as one layer of CNCs and one layer of XG (Figure 1B). Adsorption of alternating layers is entropically driven and selflimiting as a result of rinsing, and hydrogen bonding and van der Waals forces between XG and CNCs are the primary intermolecular forces holding together the films. 5,7,26 The assembled films were translucent, appearing iridescent uniformly across the entire film surface at higher thicknesses, as seen in previous studies of CNC-containing $\mathrm{LbL}$ films. ${ }^{4}$

The thickness of LbL XG-CNC films was measured by spectroscopic ellipsometry for a range of bL (and fit using a Cauchy model) to examine the evolution of film thickness as a function of the number of layers deposited. The film buildup for all variations of XG followed a linear trend with increasing number of bL (Figure 2). Linear fits for XG-CNC, USXG$\mathrm{CNC}$, and DGXG-CNC dry film thickness as a function of bL 


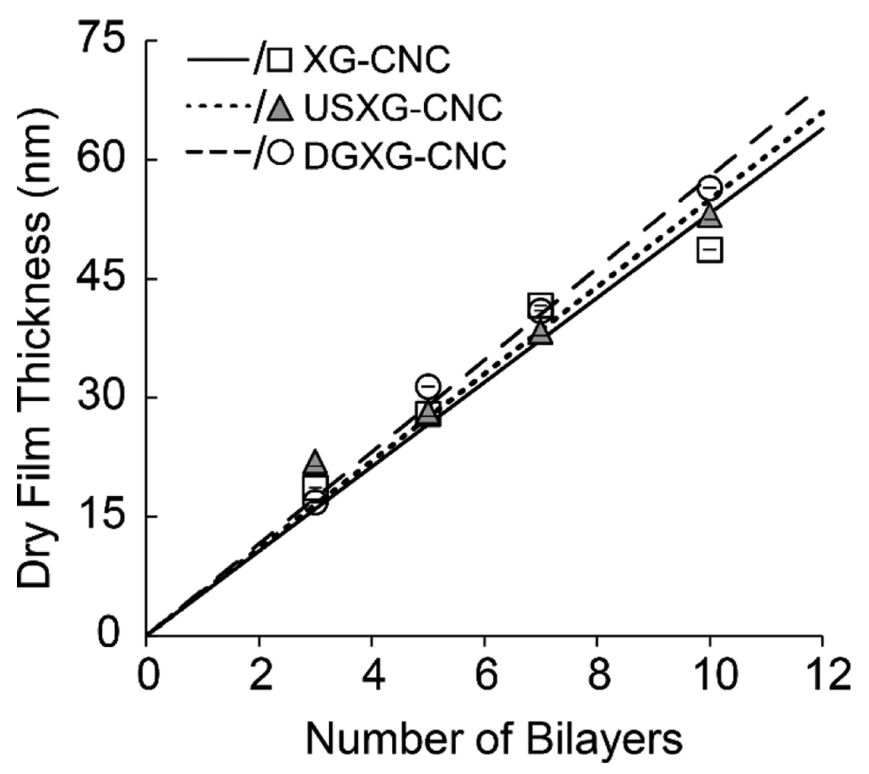

Figure 2. Plot of dry film thickness of XG/DGXG/USXG-CNC composite films as a function of the number of bL deposited. Error bars are the standard deviation of $n \geq 3$ measurements from three replicate samples and are smaller than the data symbols used.

number yielded slopes of $5.3 \pm 0.3,5.5 \pm 0.2$, and $5.8 \pm 0.1$ $\mathrm{nm} / \mathrm{bL}$, respectively. These values are within the range of those reported previously, where the growth of each bL is expected to be 5-8 nm for films with similar unmodified XG and CNC concentrations. $^{5,7,8,57}$ This highlights the reproducibility of the LbL method, even with small changes in experimental protocols between labs. While the thickness increment per bL for films with XG, USXG, and DGXG is very similar, it does not necessarily translate into identical film composition. It could imply that a different mass is adsorbed with a different conformation (but the same thickness) or that a decrease in adsorption of one component is compensated by an increase in the other. Moreover, we have observed that CNCs are the main contributors to thickness, such that changes in the amount of XG adsorbed or its conformation does not impact the overall bL thickness. ${ }^{7,8,57}$ Previously, we have shown that after $15 \mathrm{~min}$ of adsorption (the dip coating time used here) DGXG adsorbs to a CNC surface in only slightly larger amounts than $\mathrm{XG}$, as measured by quartz-crystal microbalance gravimetry; however, after $1 \mathrm{~h}$ of adsorption, there was significantly more DGXG adsorbed than XG. ${ }^{36}$ The previous adsorption data ${ }^{36}$ and compositional analysis by X-ray diffraction $^{8}$ support these ellipsometry results, suggesting that a similar composition is expected for XG-CNC films constructed with the three types of XG used in this study and that CNCs make up $>72 \%$ of the overall film by mass.

XG can either adsorb onto CNCs to form "loop and tail" or flat extended conformations, depending on the concentration ${ }^{25}$ or $\mathrm{MW}^{32}$ Despite differences in adsorbed conformation, different MW XG adsorbed onto CNCs have been shown to have similar thickness (at $0 \% \mathrm{RH}$ ), even though XG with a "loop and tail" conformation has a higher swelling capacity when tethered between CNCs, leading to thicker layers when measured in water. ${ }^{32}$ The CNCs used in this work (sulfuric acid hydrolyzed kraft pulp from CelluForce) have an average cross section of $6 \pm 2 \mathrm{~nm},{ }^{43}$ indicating that each adsorbed bL is approximately one monolayer of nanocrystals with a relatively flat XG layer that does not contribute considerably to the film thickness but is crucial to allow the subsequent layer of CNCs to be adsorbed. Previous studies imply that XG fills in spaces between randomly oriented CNCs and deposits on top of CNCs, ${ }^{58}$ leading to an overall smoother $^{8}$ and denser structure than CNCs alone would impart (and where the structure is likely somewhat stratified due to drying steps during deposition $\left.{ }^{40,59}\right)$. CNC-only films are porous with an optical density (i.e., refractive index) of $1.46,{ }^{60}$ whereas an average refractive index of 1.51 was used to fit ellipsometry data herein indicating nonporous films. Overall, the LbL films with CNCs and unmodified or modified XG were uniform with similar build-up behavior and reproducible film thickness.

Thermal Shrinking of XG-CNC Layered Thin Films. Thermal shrinking was used to characterize the mechanical properties of layered XG-CNC, USXG-CNC, and DGXGCNC films with varying thickness. In this method, films deposited onto prestressed PS were shrunk in an oven at 135 ${ }^{\circ} \mathrm{C}$-above the $T_{\mathrm{g}}$ of PS. Because of the elastic modulus mismatch between the film and substrate, shrinking of the compliant prestressed PS caused the composite film to buckle to dissipate strain, ${ }^{8}$ resulting in a wrinkled surface topography (Figure 3A). The CNCs do not appear to re-orient during

A

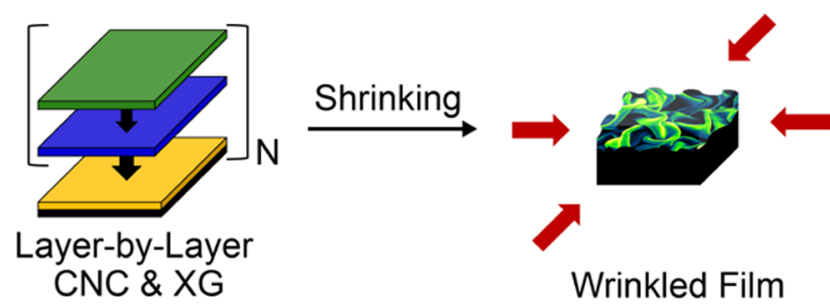

B

$3 \mathrm{bL}$
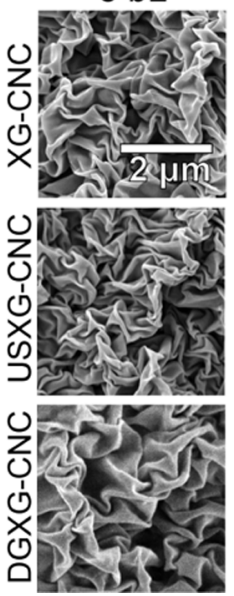

$5 \mathrm{bL}$

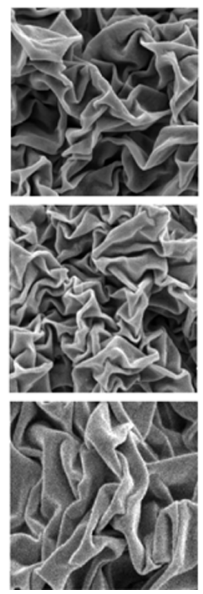

$7 \mathrm{bL}$

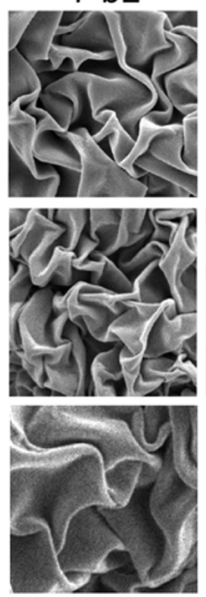

$10 \mathrm{bL}$

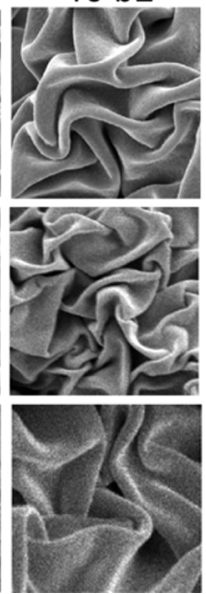

Figure 3. Thermal shrinking-induced buckling of $\mathrm{XG}-\mathrm{CNC}$ composite films. (A) Schematic representation of the shrinking process, illustrating the isotropic shrinking in the plane of the PS substrate, and (B) SEM images of XG/USXG/DGXG-CNC films after shrinking. Films of varied thickness are shown as number of bL.

shrinking and remain parallel to the wrinkled $x-y$ plane. ${ }^{47}$ The wrinkle size (or "wavelength") is characteristic of the mechanical properties as well as the thickness of the film. Shrinking at $135{ }^{\circ} \mathrm{C}$ is not expected to degrade XG or CNCs, as their thermal stability is exceptionally high (onset of degradation occurs above $300{ }^{\circ} \mathrm{C}$ for $\mathrm{XG}^{61}$ and around $275^{\circ} \mathrm{C}$ for sodium form sulfated $\mathrm{CNCs}^{62}$ ) and is also significantly 
below the $T_{\mathrm{g}}$ of XG $\left(257-275{ }^{\circ} \mathrm{C}^{63-65}\right)$, implying that the shrinking is performed when XG is in its "high modulus" glassy state. $^{65}$

Figure $3 \mathrm{~B}$ shows SEM images of the buckled unmodified and modified XG-CNC films after thermal shrinking. All films had a consistent wrinkle morphology across the surface where the wrinkle size increased with film thickness (bL number), however, not to the same extent for all film compositions. The wrinkles of DGXG-CNC films are visibly larger than XGCNC and USXG-CNC. As Figure 2 shows only small differences in film thickness at the same bL number for the films constructed with different variations of XG, the wrinkle size difference is primarily attributed to differences in mechanical properties, rather than film thickness. From a qualitative assessment of the SEM images, we can infer that DGXG-CNC films have the highest elastic modulus because of their larger wrinkles. Thus, the simple comparison of wrinkle sizes suggests that the structure of $\mathrm{XG}$ influences mechanical properties of $\mathrm{XG}-\mathrm{CNC}$ layered films which begins to lend to the understanding of structure-function relationships in the plant cell wall.

We also note that the consistent wrinkle morphology and lack of cracks or defects in the films after shrinking implies they are fairly ductile, even at a high strain of $\sim 0.6$ being employed here. Furthermore, the substrate appears to easily "flow" under the film when heated above its $T_{g}$, again with no delamination or damage to the film observed over the many samples tested.

Elastic Moduli of XG-CNC Layered Thin Films. Elastic modulus values of $\mathrm{XG}-\mathrm{CNC}$ films were calculated using the thermally induced buckling method described previously in the literature, ${ }^{8,47}$ based on quantitative image analysis of the wrinkled structures. The characteristic wrinkle wavelength of buckled films was determined by fast Fourier transform (FFT) analysis of SEM images with appropriate feature edge detection, filtering, and curve fitting. We have shown that this automated method is high throughput and provides an unbiased and accurate measurement of the periodic wrinkle sizes, as opposed to manual feature sizing techniques. ${ }^{52}$ An example of this analysis is depicted for $\mathrm{XG}-\mathrm{CNC}$, USXG$\mathrm{CNC}$, and DGXG-CNC films with $7 \mathrm{bL}$, buckled by thermal shrinking (Figure 4). The analysis progression is shown, starting from the raw SEM images (Figure 4A), to the edge detection maps (Figure 4B), to the intensity versus spatial frequency spectra (Figure 4C), and finally, the Gaussian curve fits of the relevant peaks are used to extract the characteristic wrinkle wavelength (Figure 4D). The reciprocal of the mean of each Gaussian curve fit is taken as the characteristic wavelength used to calculate the modulus.

A plot of characteristic wrinkle wavelength $(\zeta)$ versus film thickness ( $h$, from ellipsometry) for all XG-CNC film compositions is shown in Figure 5. Linear regressions were fit to $\zeta$ versus $h$ data and the slopes $(\zeta / h)$ were used to calculate the elastic modulus of each film based on eqs 1 and 2 . The obtained elastic moduli for XG-CNC, USXG-CNC, and DGXG-CNC layered films were $19 \pm 2,27 \pm 1$, and $75 \pm 6$ $\mathrm{GPa}$, respectively. Statistical analysis was performed, indicating that there is a statistically significant difference between all three modulus values $(P<0.05)$. The elastic moduli of all films fall between those measured previously for CNCs (57-143 $\mathrm{GPa}^{15,44}$ ) and XG extracted from tamarind seed $\left(5.95 \mathrm{GPa}^{65}\right.$ ) individually, which is expected because CNCs make up over $72 \%$ of the film by mass ${ }^{8}$ and are known to be mechanical reinforcing agents in polymer composites and films. ${ }^{66,67}$

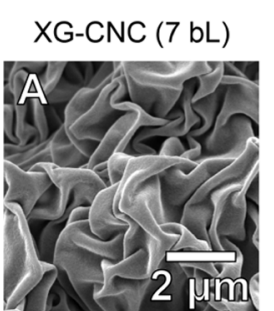

USXG-CNC (7 bL)

DGXG-CNC (7 bL)
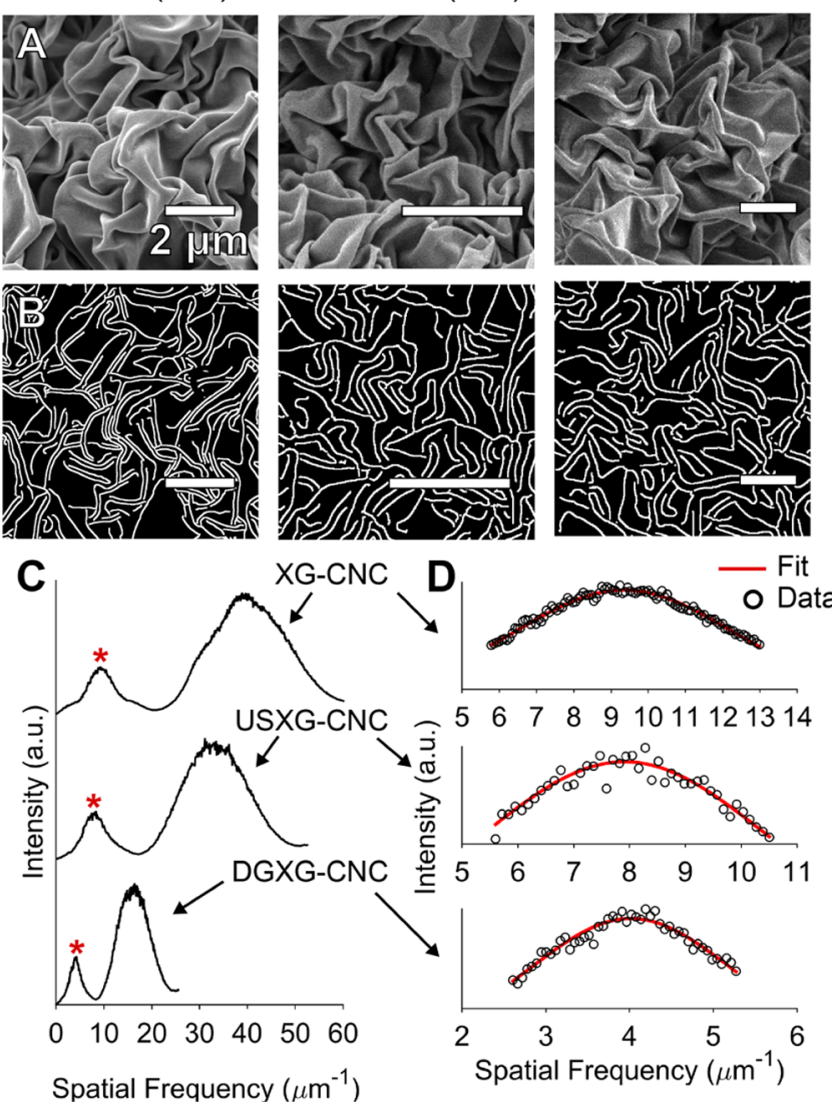

Figure 4. FFT analysis method for the determination of characteristic wrinkle wavelengths. (A) SEM images of XG-CNC (left), USXGCNC (center), and DGXG-CNC (right) films at $7 \mathrm{bL}$, with (B) their respective edge detection maps. FFT analysis is conducted on the edge detection maps in (B), to give (C) intensity vs spatial frequency plots. The characteristic wavelengths lie within the peaks identified by red asterisks in (C), which are subjected to a Gaussian curve fitting algorithm to give (D) output curve fits from which the reciprocal of the spatial frequency at the mean of the Gaussian fit is taken as the characteristic wrinkle wavelength.

Because we infer from our analysis that the film composition with the different XG types is similar, the moduli measured $(19-75 \mathrm{GPa})$ are interpreted to be primarily affected by the strength of the interactions between CNCs and XG.

There is approximately a 4-fold difference in moduli between DGXG-CNC and $\mathrm{XG}-\mathrm{CNC}$ films, whereas the elastic modulus of USXG-CNC films is only 1.4 times greater than that of XG-CNC films, indicating that the modulus is affected more by the degree of galactosylation than the MW of XG. When adsorbed on the surface of CNCs, high MW XG takes on a "loop and tail" conformation, whereas low MW XG tends to form flatter extended conformations with reduced flexibility and mobility. ${ }^{32}$ It has been shown that, when comparing CNC nanocomposite materials with low and high MW polymers of similar structure, the reduced chain mobility in shorter chain polymers leads to greater stiffness in composite materials, at least for polymers that are unbranched or have short branches. ${ }^{32,66}$ This behavior explains the higher modulus measured for USXG-CNC films compared to XGCNC films and is also consistent with the biomechanical hotspot model where extended XG chains pack between cellulose-cellulose junctions, forming "hotspots" of greater 


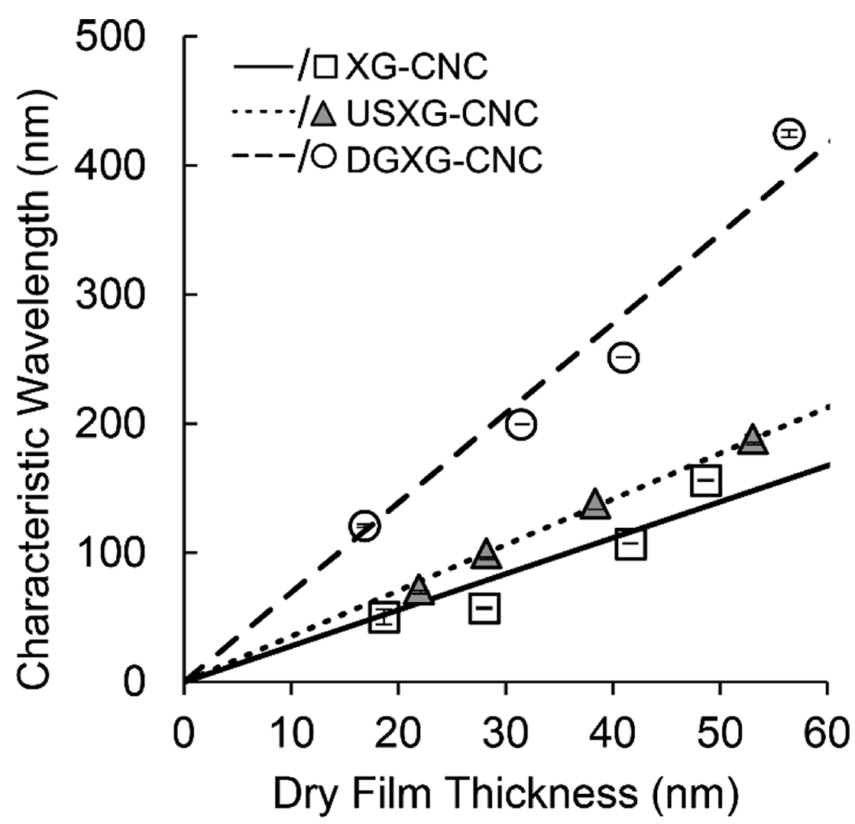

Figure 5. Plot of characteristic wavelength as a function of dry film thickness for thermally buckled XG/USXG/DGXG-CNC films. Error bars represent the standard deviation from $n \geq 3$ independently prepared sample replicates. All linear regressions were forced to go through the origin and yielded $R^{2}>0.97$.

stiffness. $^{24,68}$ Because USXG and DGXG have similar MWs (that are both lower than unmodified $X G$ ), this further emphasizes the importance of the degree of galactosylation on mechanical properties, evidenced by the almost 3-fold increase in elastic modulus between USXG-CNC and DGXG-CNC films. This is attributed to the fact that removal of galactose residues decreases the steric hindrance between adjacent DGXG chains and between DGXG and CNCs, ${ }^{30,34,35,69}$ resulting in a more tightly bound DGXG-CNC matrix than for films constructed with either unmodified XG or USXG. From these observations, we can conclude that in a dry state, the MW and degree of galactosylation both influence how XG interacts with CNCs and in turn impact the elastic modulus of the different $\mathrm{XG}-\mathrm{CNC}$ films. These findings highlight that the branched structure of XG in nature is likely required to impart flexibility (reduced stiffness) in the plant cell wall, where XG$\mathrm{CNC}$ interactions are strong but the "composite" itself does not require a high modulus for its function. Conversely, to produce higher modulus, and potentially stronger, biobased composites from cellulose and hemicelluloses, reducing saccharide residues and polymer MW may be advantageous.

Previous measurements of plant cell wall mimics have yielded a wide range of elastic modulus values, from hundreds of $\mathrm{MPa}^{55}$ to tens of $\mathrm{GPa}{ }^{8,11,65}$ The discrepancy stems from the different material compositions, sample forms (e.g., thin films, ${ }^{8}$ thick films, ${ }^{65}$ and printed structures ${ }^{11}$ ), and testing methods/ conditions (e.g., various forms of tensile testing ${ }^{55,65}$ or thermal shrinking ${ }^{8}$ ). Elastic moduli ranging from 2 to $5 \mathrm{GPa}$ were measured for a range of dehydrated plant cells, noting that these values are often much lower $(100 \mathrm{~s}$ of $\mathrm{MPa})$ for hydrated plant cells. ${ }^{10,55,70}$ Tensile tests performed on free-standing tamarind seed XG films resulted in an elastic modulus of $\sim 6$ GPa (at $0 \% \mathrm{RH}) .^{65}$ All of these values are much lower than those found in this work, primarily because of the "oversimplification" of our model films and because CNCs are known to significantly reinforce polymer matrices. ${ }^{45}$ While CNCs are a good mimic for cellulose in nature and help to construct reproducible and smooth films, they are stronger and stiffer than native cellulose because they lack the dislocations and disordered regions of the microfibril. Nonetheless, in our model films, CNCs serve the same reinforcing function as cellulose in the plant cell wall, which leads to high modulus $\mathrm{XG}-\mathrm{CNC}$ films.

Interestingly, as a result of the low density of our films (the density of cellulose and XG are very similar at ca. $1.5 \mathrm{~g} /$ $\left.\mathrm{cm}^{3}\right),{ }^{19,71}$ their specific elastic moduli suggests high-performance materials comparable to engineering polymers and metal alloys. The lower limits for the specific elastic moduli estimated for XG-CNC, USXG-CNC, and DGXG-CNC layered films were 13,18 , and $50 \mathrm{GPa} \mathrm{g}^{-1} \mathrm{~cm}^{3}$, respectively, which are generally on par with those reported for various wood species $\left(10-20 \mathrm{GPa} \mathrm{g}^{-1} \mathrm{~cm}^{3}\right)^{72}$ and significantly surpass other polymers/commodity plastics $\left(0.2-3 \mathrm{GPa} \mathrm{g}^{-1} \mathrm{~cm}^{3}\right) .^{72}$ The stiffest DGXG-CNC materials prepared herein had twice the specific modulus of lightweight alloys like steel, aluminum, and

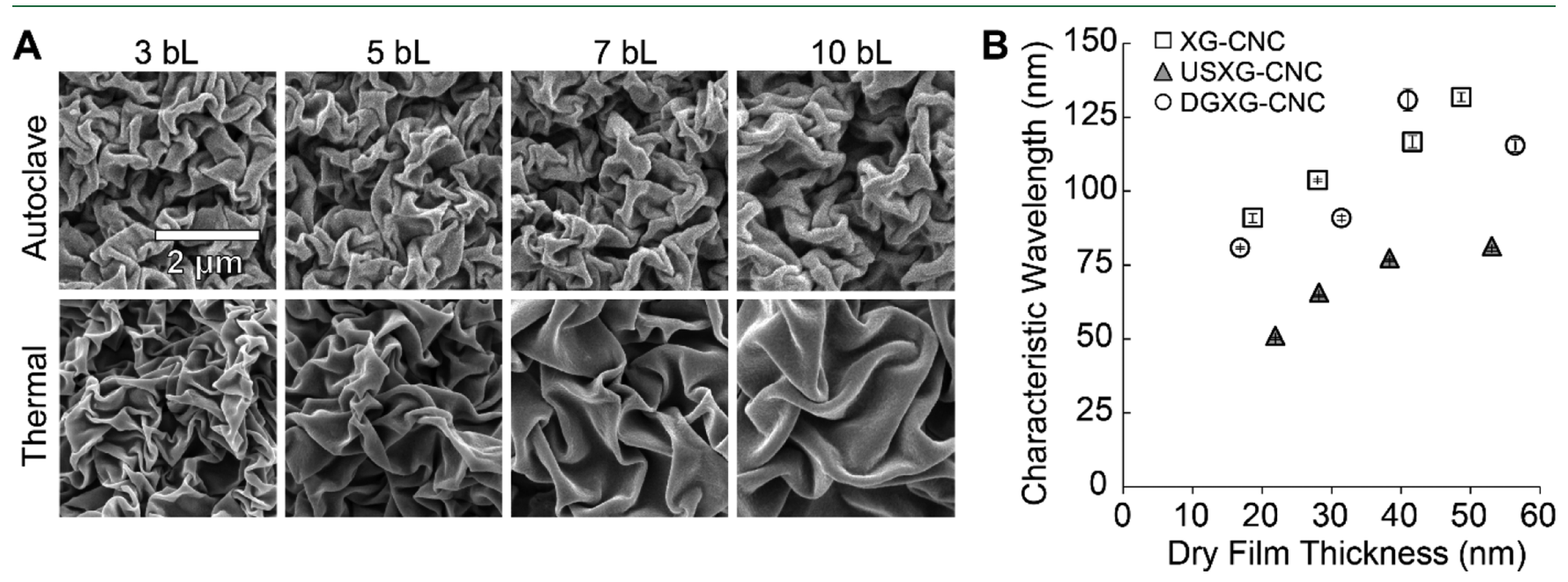

Figure 6. (A) SEM images of representative unmodified XG-CNC films after autoclave shrinking (top row) compared to thermal shrinking in an oven (bottom row). Films of varied thickness are shown as number of bL. (B) Plot of characteristic wavelength as a function of dry film thickness for autoclave-buckled XG/USXG/DGXG-CNC films. Error bars represent the standard deviation from $n \geq 3$ sample replicates. 
titanium $\left(\sim 25 \mathrm{GPa} \mathrm{g}^{-1} \mathrm{~cm}^{3}\right)^{72}$ and a specific modulus similar to Kevlar $\left(49-78 \mathrm{GPa} \mathrm{g} \mathrm{gm}^{3}\right){ }^{73}$ This supports the application of such biobased materials in a range of protective, construction, and transportation applications, where the lightweight property is a particular asset.

Buckling-based methods allow a direct (and precise) comparison of moduli between materials. ${ }^{47}$ However, they are very sensitive to the way the characteristic wrinkle length is defined and how the method is calibrated. ${ }^{47}$ This has led to significant differences in the values previously reported $(70 \pm 2$ $\left.\mathrm{GPa}^{8}\right)$ versus those obtained here $(19 \pm 2 \mathrm{GPa})$ for the elastic modulus of unmodified XG-CNC films, using the same thermal shrinking method. The wrinkle wavelength is extracted from the Fourier analysis, which also depends on the capacity of edge detection and user interpretation of the "wrinkle" that is being measured. ${ }^{47,52}$ Therefore, the discrepancy between the measured moduli in this work and those determined previously on similar materials is mainly attributed to image analysis, and to a lesser degree, to the differences in the XG structure/ properties between studies. In fact, these differences could be reconciled if the same image analysis procedure (shown in Figure 4) was carried out for previously obtained SEM images (i.e., a modulus of $20 \pm 4 \mathrm{GPa}$ is calculated for XG-CNC films after analyzing data from ref 8 with the algorithm used in this study). This indicates that buckling-based methods are highly effective for relative modulus comparison between materials within the same study, but to ensure accurate and consistent values across studies, the same (robust) data analysis treatment must be used. ${ }^{47}$

Shrinking of Hydrated XG-CNC Films. To understand the effect of hydration on the mechanical properties of XGCNC films, the composite films were shrunk and fully hydrated in an autoclave at $121{ }^{\circ} \mathrm{C}$. To the best of our knowledge, this is the first time an autoclave shrinking approach is used to buckle "wet" thin films. After shrinking, the samples were dried and wrinkle morphology was imaged by SEM (Figure 6A), which revealed that the films shrunk while hydrated had smaller wrinkles than those shrunk in an oven under dry conditions. Similar to Figure 3, the wrinkle size increased with increasing number of bL, and these changes can be quantified through FFT image analysis. A plot of the characteristic wavelength of films buckled in an autoclave as a function of dry film thickness for $\mathrm{XG}-\mathrm{CNC}$, USXG-CNC, and $\mathrm{DGXG}-\mathrm{CNC}$ films is shown in Figure 6B. While the data show that characteristic wavelength increases with dry film thickness, the trend is not linear and the fitted lines do not adjust well to the data, with $R^{2}$ values $<0.95$. Therefore, a plot of characteristic wavelength versus dry film thickness does not allow the direct calculation of elastic moduli using eq 2 . This result is not completely unexpected, as these highly hygroscopic films should remain fully hydrated as they buckled in the autoclave. While it is anticipated that hydrated films would be thicker because of swelling, ${ }^{32}$ we could not measure the hydrated film thicknesses because spectroscopic ellipsometry could not be conducted under conditions that approximate the level of hydration in the autoclave. Nevertheless, when comparing the images obtained from the hydrated and dry shrinking methods, where hydrated films would be thicker than their dry counterparts, the fact that we observe smaller wrinkles implies a significantly lower elastic modulus for the (expectedly softer) hydrated films.

In an effort to calculate an approximate modulus for the hydrated films, we used the change in thickness reported in a study that compared dry and hydrated XG films of different
MW (low MW USXG and unmodified tamarind XG). ${ }^{32}$ In that study, it was found that films made of unmodified XG and CNCs doubled in thickness when hydrated, whereas films made of low MW XG and CNCs showed no significant change in thickness. This behavior was ascribed to the greater swelling capacity of higher MW XG, which forms a more loopy structure than USXG between CNC layers. Given that our unmodified XG, USXG, and CNCs are similar to those used in this previous study, we can assume that the materials used herein would behave similarly. Following this logic, the thickness of USXG-CNC films would remain largely unchanged, while $\mathrm{XG}-\mathrm{CNC}$ films would be twice as thick when hydrated. ${ }^{32}$ Therefore, the XG-CNC data in Figure 6B would be shifted to the right along the $x$-axis and have a shallower slope, while the USXG-CNC data would remain the same (Supporting Information, Figure S1). If this adjusted data are used to calculate the elastic modulus, it yields values of $8 \pm$ 1 and $10 \pm 2 \mathrm{GPa}$, for XG-CNC and USXG-CNC films, respectively (using $\zeta / h$ slopes based on linear regressions fit through a 0 intercept, with $\left.R^{2}>0.96\right)$. These values are approximately $60 \%$ lower than the corresponding modulus values of dry films measured by thermal shrinking in an oven. This is in accordance with reports for films of tamarind seed $\mathrm{XG}$ only, which gave a $\sim 70 \%$ reduction in modulus when measured at $0 \% \mathrm{RH}$ compared to $99 \% \mathrm{RH}$ (5.9 \pm 0.8 and 1.6 $\pm 0.3 \mathrm{GPa}$, respectively). ${ }^{65}$

We conclude that the XG-CNC films soften when hydrated, from qualitative analysis of SEM images, and estimated moduli based on previously reported film thickness changes. While hydrated USXG-CNC films appear stiffer than XG-CNC because of less film swelling, exact film thickness quantification is needed to confirm this. This also supports that lower MW XG gives stiffer films under both dry and hydrated conditions. Overall, the difference in moduli between film types is smaller when the films are wet, and, for example, hydrated DGXG-CNC film wavelength data are very similar to $\mathrm{XG}-\mathrm{CNC}$ films, indicating a similar softening behavior. Unfortunately, we cannot estimate a modulus for DGXGCNC films because the film thickness/swelling capacity of DGXG-CNC films has yet to be explored. Because living plant cell walls are normally hydrated, these findings imply that the cell wall is less stiff, or more elastic, in a hydrated state than when dry, which allows the plant cell to expand and contract depending on environmental conditions and needs during growth cycles. In addition to providing qualitative information about hydrated film mechanical properties (which is important for hygroscopic materials that are rarely fully dried and materials that are used in their wet state), autoclave shrinking could be useful for tailoring interfacial properties such as specific surface area, hydrophobicity, and stretchability for materials that must remain hydrated at all times to maintain their internal structure/properties such as hydrogels, biomaterials, underwater adhesives, and so forth.

\section{CONCLUSIONS}

In this work, we have used a thermal shrinking buckling-based method to compare the elastic moduli of XG-CNC thin films constructed with unmodified, low MW, and partially DGXG. From thermal (oven) shrinking, the elastic modulus of XGCNC $(19 \pm 2 \mathrm{GPa})$ was determined to be lower than that of USXG-CNC $(27 \pm 1 \mathrm{GPa})$ and much lower than that of DGXG-CNC $(75 \pm 6 \mathrm{GPa})$. We infer that the conformational changes of XG based on MW lead to the higher modulus of 
USXG-CNC films because of its flat, compact adsorbed conformation versus "loops and tails" conformation of higher MW XG. The even greater modulus exhibited by DGXGCNC films indicates that a higher binding capacity to CNCs for XG with a reduced degree of galactosylation, coupled with potentially flatter adsorbed conformation because of reduced steric hindrance and MW, strongly influence mechanical properties, resulting in stiffer composite materials. Overall, the films are highly ductile and do not crack or delaminate under any of the experimental conditions tested. We have also presented, for the first time, autoclave shrinking of films to mimic a hydrated environment during film buckling. The qualitative analysis of $\mathrm{XG}-\mathrm{CNC}$ films buckled using autoclave shrinking suggests that $\mathrm{XG}-\mathrm{CNC}$ films soften when hydrated, reducing the elastic modulus by approximately $60 \%$ compared to dry conditions. We can extrapolate these findings to the plant cell wall, concluding that the MW and saccharide residue composition of XG impact the overall flexibility and rigidity of the plant cell, where more interactions (from reduced steric hindrance of DGXG and/or flatter conformations of USXG) between $\mathrm{XG}$ and $\mathrm{CNCs}$ result in higher elastic modulus materials.

This work also provides useful insights into how structural changes in XG affect the mechanical properties of composite materials, which can be used to design biobased materials from cellulose and hemicelluloses with tunable properties. Based on this work, we suggest that to make a stiffer material with a higher elastic modulus, the degree of galactosylation of XG should be reduced, and/or a low MW XG should be used. Additionally, because a higher modulus was observed because of the modifications which increased interactions between XG and CNCs or formed a more compact structure, alternative modifications that achieve similar structural variations should effectively increase elastic modulus. In contrast, a higher MW XG with a high substitution of saccharide residues would be useful for fabricating a material that is more elastic. These design suggestions would be useful to develop lightweight biobased materials for applications in biosensing, packaging, protective materials, transportation paneling, drug delivery, and tissue engineering scaffolds, to name just a few.

\section{ASSOCIATED CONTENT}

\section{(s) Supporting Information}

The Supporting Information is available free of charge at https://pubs.acs.org/doi/10.1021/acs.biomac.0c01031.

Plot of characteristic wavelength versus assumed hydrated film thickness for XG-CNC and USXGCNC films (PDF)

\section{AUTHOR INFORMATION}

\section{Corresponding Authors}

Jose M. Moran-Mirabal - Department of Chemistry and Chemical Biology, McMaster University, Hamilton, Ontario L8S 4M1, Canada; (ㅇ orcid.org/0000-0002-4811-3085; Phone: (905) 525-9140 ext. 24507; Email: mirabj@ mcmaster.ca

Emily D. Cranston - Department of Chemical Engineering, McMaster University, Hamilton, Ontario L8S 4L7, Canada; Department of Wood Science and Department of Chemical and Biological Engineering, The University of British Columbia, Vancouver, British Columbia V6T 1Z4, Canada; 이이.org/
0000-0003-4210-9787; Phone: 604-827-0627;

Email: emily.cranston@ubc.ca

\section{Authors}

Taylor C. Stimpson - Department of Chemical Engineering, McMaster University, Hamilton, Ontario L8S 4L7, Canada

Bernard Cathala - INRAE, UR BIA, Nantes F-44316, France; (1) orcid.org/0000-0002-3844-872X

Céline Moreau - INRAE, UR BIA, Nantes F-44316, France

Complete contact information is available at:

https://pubs.acs.org/10.1021/acs.biomac.0c01031

\section{Author Contributions}

The manuscript was written through contributions of all authors. All authors have given approval to the final version of the manuscript.

\section{Funding}

Natural Sciences and Engineering Research Council of Canada (Discovery Grants RGPIN 2019-06433 and 05252). Labex Serenade program (no. ANR-11-LABX-0064) funded by the "Investissements d'Avenir" program of the French National Research Agency (ANR) through the AMIDEX project (no. ANR-11-IDEX-0001-02).

\section{Notes}

The authors declare no competing financial interest.

\section{ACKNOWLEDGMENTS}

The authors acknowledge the researchers and facilities of the Biopolymères, Interactions, et Assemblages at the Institut National de Recherche pour l'Agriculture, l'Alimentation et l'Environnement (INRAE) in Nantes, France, for their collaboration on this work. Specifically, we would like to thank L. Saulnier (HPSEC), K. Cahier (HPSEC), and A. Gourlay (XG fractionation) at INRAE. We also thank D. Osorio for acquiring SEM images, D. Wagner for assistance in MATLAB algorithm development for FFT analysis, and S. Saem for sputtering of gold films (all at McMaster University). We gratefully acknowledge useful discussions with Prof. M. Rheinstadter (Department of Physics and Astronomy, McMaster) regarding film composition and architecture. J.M.M.-M. and E.D.C. are recipients of Early Researcher awards from the Ontario Ministry of Research and Innovation. J.M.M.-M. is the Tier 2 Canada Research Chair in Micro and Nanostructured Materials, and E.D.C. held the Tier 2 Canada Research Chair in Bio-Based Nanomaterials and is currently the UBC President's Excellence Chair in Forest Bioproducts. T.C.S. is the recipient of a Queen Elizabeth II Ontario Graduate Scholarship. The research presented herein was supported by funding through the Natural Sciences and Engineering Research Council of Canada and made use of facilities at the Canadian Centre for Electron Microscopy (CCEM) and the McMaster Centre for Emerging Device Technologies (CEDT). This work is a contribution to the Labex Serenade program (no. ANR-11-LABX-0064) funded by the "Investissements d'Avenir" program of the French National Research Agency (ANR) through the AMIDEX project (no. ANR-11-IDEX-0001-02).

\section{ABBREVIATIONS}

XG, xyloglucan; CNC, cellulose nanocrystal; MW, molecular weight; PEI, polyethylene imine; PS, polystyrene; $T_{\mathrm{g}}$, glass transition temperature; LbL, layer-by-layer; $\mathrm{PAH}$, polyallyl- 
amine hydrochloride; USXG, ultrasonicated xyloglucan; DGXG, degalactosylated xyloglucan; bL, bilayer; HPSEC, high-performance size exclusion chromatography; SEM, scanning electron microscopy; $\mathrm{RH}$, relative humidity; FFT, fast Fourier transform

\section{REFERENCES}

(1) Albersheim, P.; An, J.; Freshour, G.; Fuller, M. S.; Guillen, R.; Ham, K.-S.; Hahn, M. G.; Huang, J.; O'Neill, M.; Whitcombe, A.; et al. Structure and Function Studies of Plant Cell Wall Polysaccharides. Biochem. Soc. Trans. 1994, 22, 374-378.

(2) Keegstra, K. Plant Cell Walls. Plant Physiol. 2010, 154, 483-486.

(3) Burgert, I. Exploring the Micromechanical Design of Plant Cell Walls. Am. J. Bot. 2006, 93, 1391-1401.

(4) Martin, C.; Jean, B. Nanocellulose/Polymer Multilayered Thin Films: Tunable Architectures towards Tailored Physical Properties. Nord. Pulp Pap. Res. J. 2014, 29, 19-30.

(5) Jean, B.; Heux, L.; Dubreuil, F.; Chambat, G.; Cousin, F. NonElectrostatic Building of Biomimetic Cellulose-Xyloglucan Multilayers. Langmuir 2009, 25, 3920-3923.

(6) Guyomard-Lack, A.; Cerclier, C.; Beury, N.; Jean, B.; Cousin, F.; Moreau, C.; Cathala, B. Nano-Structured Cellulose NanocrystalsXyloglucan Multilayered Films for the Detection of Cellulase Activity. Eur. Phys. J.: Spec. Top. 2012, 213, 291-294.

(7) Cerclier, C. V.; Guyomard-Lack, A.; Cousin, F.; Jean, B.; Bonnin, E.; Cathala, B.; Moreau, C. Xyloglucan-Cellulose Nanocrystal Multilayered Films: Effect of Film Architecture on Enzymatic Hydrolysis. Biomacromolecules 2013, 14, 3599-3609.

(8) Gill, U.; Sutherland, T.; Himbert, S.; Zhu, Y.; Rheinstädter, M. C.; Cranston, E. D.; Moran-Mirabal, J. M. Beyond Buckling: Humidity-Independent Measurement of the Mechanical Properties of Green Nanobiocomposite Films. Nanoscale 2017, 9, 7781-7790.

(9) Cybulska, J.; Vanstreels, E.; Ho, Q. T.; Courtin, C. M.; Van Craeyveld, V.; Nicolaï, B.; Zdunek, A.; Konstankiewicz, K. Mechanical Characteristics of Artificial Cell Walls. J. Food Eng. 2010, 96, 287294.

(10) Bidhendi, A. J.; Geitmann, A. Methods to Quantify Primary Plant Cell Wall Mechanics. J. Exp. Bot. 2019, 70, 3615-3648.

(11) Malek, S.; Raney, J. R.; Lewis, J. A.; Gibson, L. J. Lightweight 3D Cellular Composites Inspired by Balsa. Bioinspiration Biomimetics 2017, 12, 026014.

(12) Mackay, A. L.; Wallace, J. C.; Sasaki, K.; Taylor, I. E. P. Investigation of the Physical Structure of the Primary Plant Cell Wall by Proton Magnetic Resonance. Biochemistry 1988, 27, 1467-1473.

(13) Varner, J. E.; Lin, L.-S. Plant Cell Wall Architecture. Cell 1989, 56, 231-239.

(14) Somerville, C. Cellulose Biosynthesis in Higher Plants. Annu. Rev. Cell Dev. Biol. 2006, 22, 53-78.

(15) Mariano, M.; El Kissi, N.; Dufresne, A. Cellulose Nanocrystals and Related Nanocomposites: Review of Some Properties and Challenges. J. Polym. Sci., Part B: Polym. Phys. 2014, 52, 791-806.

(16) Rose, M.; Babi, M.; Moran-Mirabal, J. The Study of Cellulose Structure and Depolymerization through Single-Molecule Methods. Ind. Biotechnol. 2015, 11, 16-24.

(17) Aulin, C.; Johansson, E.; WÅgberg, L.; Lindström, T. SelfOrganized Films from Cellulose I Nanofibrils Using the Layer-byLayer Technique. Biomacromolecules 2010, 11, 872-882.

(18) Scheller, H. V.; Ulvskov, P. Hemicelluloses. Annu. Rev. Plant Biol. 2010, 61, 263-289.

(19) Aulin, C.; Gällstedt, M.; Lindström, T. Oxygen and Oil Properties of Microfibrillated Cellulose Films and Coatings. Cellulose 2010, 17, 559-574.

(20) Zabotina, O. A. Xyloglucan and Its Biosynthesis. Front. Plant Sci. 2012, 3, 134.

(21) Fry, S. C. The Structure and Functions of Xyloglucan. J. Exp. Bot. 1989, 40, 1-11.

(22) Eckardt, N. A. Role of Xyloglucan in Primary Cell Walls. Plant Cell 2008, 20, 1421-1422.
(23) Hanus, J.; Mazeau, K. The Xyloglucan-Cellulose Assembly at the Atomic Scale. Biopolymers 2006, 82, 59-73.

(24) Park, Y. B.; Cosgrove, D. J. Xyloglucan and Its Interactions with Other Components of the Growing Cell Wall. Plant Cell Physiol. 2015, 56, 180-194.

(25) Villares, A.; Moreau, C.; Dammak, A.; Capron, I.; Cathala, B. Kinetic Aspects of the Adsorption of Xyloglucan onto Cellulose Nanocrystals. Soft Matter 2015, 11, 6472-6481.

(26) Benselfelt, T.; Cranston, E. D.; Ondaral, S.; Johansson, E.; Brumer, H.; Rutland, M. W.; WÅgberg, L. Adsorption of Xyloglucan onto Cellulose Surfaces of Different Morphologies: An EntropyDriven Process. Biomacromolecules 2016, 17, 2801-2811.

(27) Park, Y. B.; Cosgrove, D. J. Xyloglucan and Its Interactions with Other Components of the Growing Cell Wall. Plant Cell Physiol. 2015, 56, 180-194.

(28) Dammak, A.; Quémener, B.; Bonnin, E.; Alvarado, C.; Bouchet, B.; Villares, A.; Moreau, C.; Cathala, B. Exploring Architecture of Xyloglucan Cellulose Nanocrystal Complexes through Enzyme Susceptibility at Different Adsorption Regimes. Biomacromolecules 2015, 16, 589-596.

(29) Lima, D. U.; Loh, W.; Buckeridge, M. S. Xyloglucan-Cellulose Interaction Depends on the Sidechains and Molecular Weight of Xyloglucan. Plant Physiol. Biochem. 2004, 42, 389-394.

(30) Whitney, S. E. C.; Wilson, E.; Webster, J.; Bacic, A.; Reid, J. S. G.; Gidley, M. J. Effects of Structural Variation in Xyloglucan Polymers on Interactions with Bacterial Cellulose. Am. J. Bot. 2006, 93, 1402-1414.

(31) Nisbet, D. R.; Crompton, K. E.; Hamilton, S. D.; Shirakawa, S.; Prankerd, R. J.; Finkelstein, D. I.; Horne, M. K.; Forsythe, J. S. Morphology and Gelation of Thermosensitive Xyloglucan Hydrogels. Biophys. Chem. 2006, 121, 14-20.

(32) Villares, A.; Bizot, H.; Moreau, C.; Rolland-Sabaté, A.; Cathala, B. Effect of Xyloglucan Molar Mass on Its Assembly onto the Cellulose Surface and Its Enzymatic Susceptibility. Carbohydr. Polym. 2017, 157, 1105-1112.

(33) Talantikite, M.; Gourlay, A.; Le Gall, S.; Cathala, B. Influence of Xyloglucan Molar Mass on Rheological Properties of Cellulose Nanocrystal/Xyloglucan Hydrogels. J. Renewable Mater. 2019, 7, $1381-1390$.

(34) de Lima, D. U.; Buckeridge, M. S. Interaction between Cellulose and Storage Xyloglucans: The Influence of the Degree of Galactosylation. Carbohydr. Polym. 2001, 46, 157-163.

(35) Brun-Graeppi, A. K. A. S.; Richard, C.; Bessodes, M.; Scherman, D.; Narita, T.; Ducouret, G.; Merten, O. W. Study on the Sol-Gel Transition of Xyloglucan Hydrogels. Carbohydr. Polym. 2010, 80, 555-562.

(36) Talantikite, M.; Stimpson, T. C.; Goulay, A.; Le-Gall, S.; Moreau, C.; Leray, N.; Cranston, E. D.; Moran-Mirabal, J. M.; Cathala, B. Bioinspired Thermo-Responsive Xyloglucan-Cellulose Nanocrystals Hydrogels. 2020, ChemRxiv. Preprint.

(37) Aulin, C.; Ahola, S.; Josefsson, P.; Nishino, T.; Hirose, Y.; Österberg, M.; WÅgberg, L. Nanoscale Cellulose Films with Different Crystallinities and Mesostructures; Their Surface Properties and Interaction with Water. Langmuir 2009, 25, 7675-7685.

(38) Roman, M. Model Cellulosic Surfaces: History and Recent Advances. Model Cellulosic Surfaces: History and Recent Advances; ACS Symposium Series; ACS, 2010; pp 3-53.

(39) WÅgberg, L.; Decher, G.; Norgren, M.; Lindström, T.; Ankerfors, M.; Axnäs, K. The Build-up of Polyelectrolyte Multilayers of Microfibrillated Cellulose and Cationic Polyelectrolytes. Langmuir 2008, 24, 784-795.

(40) Jean, B.; Dubreuil, F.; Heux, L.; Cousin, F. Structural Details of Cellulose Nanocrystals/Polyelectrolytes Multilayers Probed by Neutron Reflectivity and AFM. Langmuir 2008, 24, 3452-3458.

(41) Cerclier, C.; Cousin, F.; Bizot, H.; Moreau, C.; Cathala, B. Elaboration of Spin-Coated Cellulose-Xyloglucan Multilayered Thin Films. Langmuir 2010, 26, 17248-17255. 
(42) Vanderfleet, O. M.; Cranston, E. D. Production Routes to Tailor the Performance of Cellulose Nanocrystals. Nat. Rev. Mater. 2020, in press.

(43) Reid, M. S.; Villalobos, M.; Cranston, E. D. Benchmarking Cellulose Nanocrystals: From the Laboratory to Industrial Production. Langmuir 2017, 33, 1583-1598.

(44) Habibi, Y.; Lucia, L. A.; Rojas, O. J. Cellulose Nanocrystals: Chemistry, Self-Assembly, and Applications. Chem. Rev. 2010, 110, 3479-3500.

(45) Moon, R. J.; Martini, A.; Nairn, J.; Simonsen, J.; Youngblood, J. Cellulose Nanomaterials Review: Structure, Properties and Nanocomposites. Chem. Soc. Rev. 2011, 40, 3941-3994.

(46) Kontturi, E.; Tammelin, T.; Österberg, M. Cellulose - Model Films and the Fundamental Approach. Chem. Soc. Rev. 2006, 35, 1287-1304.

(47) Stimpson, T. C.; Osorio, D. A.; Cranston, E. D.; MoranMirabal, J. M. Elastic Modulus Determination for Nanobiocomposite Thin Films: Direct Comparison of Three Buckling-Based Methods. 2020, ChemRxiv. Preprint.

(48) Decher, G.; Schlenoff, J. B. Multilayer Thin Films: Sequential Assembly of Nanocomposite Materials; John Wiley \& Sons, 2006.

(49) Foster, E. J.; Moon, R. J.; Agarwal, U. P.; Bortner, M. J.; Bras, J.; Camarero-Espinosa, S.; Chan, K. J.; Clift, M. J. D.; Cranston, E. D.; Eichhorn, S. J.; et al. Current Characterization Methods for Cellulose Nanomaterials. Chem. Soc. Rev. 2018, 47, 2609-2679.

(50) Hodge, T. C.; Bidstrup-allen, S. A.; Kohl, P. A. Stresses in Thin Film Metallization. IEEE Trans. Compon., Packag., Manuf. Technol., Part A 1997, 20, 241-250.

(51) Canny, J. A Computational Approach to Edge Detection. IEEE Trans. Pattern Anal. Mach. Intell 1986, 8, 679-698.

(52) Stimpson, T. C.; Wagner, D. L.; Cranston, E. D.; MoranMirabal, J. M. Image Analysis of Structured Surfaces for Quantitative Topographical Characterization. 2020, ChemRxiv. Preprint.

(53) Greco, F.; Bellacicca, A.; Gemmi, M.; Cappello, V.; Mattoli, V.; Milani, P. Conducting Shrinkable Nanocomposite Based on AuNanoparticle Implanted Plastic Sheet: Tunable Thermally Induced Surface Wrinkling. ACS Appl. Mater. Interfaces 2015, 7, 7060-7065.

(54) Mott, P. H.; Dorgan, J. R.; Roland, C. M. The Bulk Modulus and Poisson's Ratio of "Incompressible" Materials. J. Sound Vib. 2008, 312, 572-575.

(55) Chanliaud, E.; Burrows, K.; Jeronimidis, G.; Gidley, M. Mechanical Properties of Primary Plant Cell Wall Analogues. Planta 2002, 215, 989-996.

(56) Decher, G. Fuzzy Nanoassemblies: Toward Layered Polymeric Multicomposites. Science 1997, 277, 1232-1237.

(57) Dammak, A.; Moreau, C.; Azzam, F.; Jean, B.; Cousin, F.; Cathala, B. Influence of Cellulose Nanocrystals Concentration and Ionic Strength on the Elaboration of Cellulose NanocrystalsXyloglucan Multilayered Thin Films. J. Colloid Interface Sci. 2015, 460, 214-220.

(58) Jean, B.; Heux, L.; Dubreuil, F.; Chambat, G.; Cousin, F. NonElectrostatic Building of Biomimetic Cellulose-Xyloglucan Multilayers. Langmuir 2009, 25, 3920-3923.

(59) Martin, C.; Barker, R.; Watkins, E. B.; Dubreuil, F.; Cranston, E. D.; Heux, L.; Jean, B. Structural Variations in Hybrid AllNanoparticle Gibbsite Nanoplatelet/Cellulose Nanocrystal Multilayered Films. Langmuir 2017, 33, 7896-7907.

(60) Reid, M. S.; Villalobos, M.; Cranston, E. D. Cellulose Nanocrystal Interactions Probed by Thin Film Swelling to Predict Dispersibility. Nanoscale 2016, 8, 12247-12257.

(61) Bergström, E. M.; Salmén, L.; Kochumalayil, J.; Berglund, L. Plasticized Xyloglucan for Improved Toughness - Thermal and Mechanical Behaviour. Carbohydr. Polym. 2012, 87, 2532-2537.

(62) Vanderfleet, O. M.; Reid, M. S.; Bras, J.; Heux, L.; GodoyVargas, J.; Panga, M. K. R.; Cranston, E. D. Insight into Thermal Stability of Cellulose Nanocrystals from New Hydrolysis Methods with Acid Blends. Cellulose 2019, 26, 507-528.

(63) Marais, A.; Kochumalayil, J. J.; Nilsson, C.; Fogelström, L.; Gamstedt, E. K. Toward an Alternative Compatibilizer for PLA/
Cellulose Composites: Grafting of Xyloglucan with PLA. Carbohydr. Polym. 2012, 89, 1038-1043.

(64) Kochumalayil, J. J.; Zhou, Q.; Kasai, W.; Berglund, L. A. Regioselective Modification of a Xyloglucan Hemicellulose for HighPerformance Biopolymer Barrier Films. Carbohydr. Polym. 2013, 93, 466-472.

(65) Kochumalayil, J.; Sehaqui, H.; Zhou, Q.; Berglund, L. A. Tamarind Seed Xyloglucan - a Thermostable High-Performance Biopolymer from Non-Food Feedstock. J. Mater. Chem. 2010, 20, 4321-4327.

(66) Reid, M. S.; Stimpson, T. C.; Niinivaara, E.; Villalobos, M.; Cranston, E. D. Comparing Soft Semicrystalline Polymer Nanocomposites Reinforced with Cellulose Nanocrystals and Fumed Silica. Ind. Eng. Chem. Res. 2018, 57, 220-230.

(67) Kargarzadeh, H.; Mariano, M.; Huang, J.; Lin, N.; Ahmad, I.; Dufresne, A.; Thomas, S. Recent Developments on Nanocellulose Reinforced Polymer Nanocomposites: A Review. Polymer 2017, 132, 368-393.

(68) Cosgrove, D. J. Re-Constructing Our Models of Cellulose and Primary Cell Wall Assembly. Curr. Opin. Plant Biol. 2014, 22, 122131.

(69) Novak Sakakibara, C.; Rita Sierakowski, M.; Chassenieux, C.; Nicolai, T.; Alves, R.; Freitas, D. Xyloglucan Gelation Induced by Enzymatic Degalactosylation; Kinetics and the Effect of the Molar Mass. Carbohydr. Polym. 2017, 174, 517-523.

(70) Wang, C. X.; Wang, L.; Thomas, C. R. Modelling the Mechanical Properties of Single Suspension-Cultured Tomato. Ann. Bot. 2004, 93, 443-453.

(71) Kochumalayil, J. Xyloglucan-Based Polymers and Nanocomposites - Modification, Properties and Barrier Film Applications. Ph.D. Thesis, KTH Royal Institute of Technology, 2012.

(72) Ashby, M. F. Materials Selection in Mechanical Design, 3rd ed.; Elsevier, 2005.

(73) DuPont. Kevlar Aramid Fiber Technical Guide, 2019 (Technical Report). 